\title{
CONFERENCIA CLÍNICO-PATOLÓGICA
}

\section{Pancreatitis aguda, adenopatías mesentéricas y diarrea}

\author{
Y. Sánchez, C. Garfia, G. López Alonsoํ, C. Yela, M. Amo, C. Arribas, F. Cruz Vigo² \\ y J. A. Solís Herruzo \\ Servicios de Medicina del Aparato Digestivo, ${ }^{1}$ Anatomía Patológica y ${ }^{2}$ Cirugía General A. Hospital Universitario 12 de \\ Octubre. Madrid
}

Sánchez Y, Garfia C, López Alonso G, Yela C, Amo M, Arribas $C$, Cruz Vigo F, Solís Herruzo JA. Pancreatitis aguda, adenopatías mesentéricas y diarrea. Rev Esp Enferm Dig 2008; 100: 779-787.

\section{CASO CLÍNICO}

Dra. Cristina Garfia. Mujer de 34 años de edad, con síndrome de Down pero adecuado desarrollo de sus capacidades motrices, cognitivas y adaptativas que acudió al Servicio de Urgencias del hospital por presentar epigastralgia intensa de 24 horas de evolución, mal definida, pero continua, sin atenuaciones ni exacerbaciones, irradiada hacia la espalda, acompañada de náuseas, vómitos biliosos y astenia. Entre sus antecedentes personales no figuraban alergias ni hábitos tóxicos y únicamente se le había realizado tres años antes una laparotomía con el fin de extirparle un "quiste de chocolate" en el ovario izquierdo. En la anamnesis por órganos y aparatos destacaba que desde la juventud presentaba síntomas dispépticos de forma intermitente, sin precisar desencadenantes, consistentes en plenitud postprandial, pirosis, molestias epigástricas inespecíficas y flatulencia. Igualmente refería episodios intermitentes y autolimitados de diarrea líquida sin productos patológicos que alternaban con fases de ritmo intestinal normal.

Recibido: 12-07-08.

Aceptado: 18-08-08.

Correspondencia: C. Garfia. Servicio de Medicina del Aparato Digestivo. Hospital Universitario 12 de Octubre. 28041 Madrid. e-mail: cgarfia@ hotmail.com
La exploración física mostraba los cambios fenotípicos propios a la trisomía 21; auscultación cardiopulmonar normal; a nivel abdominal encontramos un abdomen blando, doloroso a la palpación profunda en epigastrio sin defensa ni otros signos de irritación peritoneal; no masas ni visceromegalias palpables, ruidos hidroaéreos normales. El resto de la exploración sin alteraciones.

Las pruebas de laboratorio mostraron que el hemograma y el estudio de la coagulación realizados en el Servicio de Urgencias eran normales, pero en el análisis bioquímico de sangre destacaba que la cifra de amilasa se encontraba elevada (660 UI/l). Fue ingresada con el diagnóstico de pancreatitis aguda.

La evolución clínica de la paciente fue favorable. A las 48 horas de su ingreso, la paciente se encontraba asintomática, toleraba la ingesta de líquidos y carecía de criterios Ranson de gravedad tanto a su ingreso como a las 48 horas. Durante su ingreso se realizaron los siguientes estudios analíticos: hemograma, coagulación, función renal y hepática y lípidos que fueron todos normales. La amilasa sérica era de $624 \mathrm{UI} / 1$ y la lipasa $8.859 \mathrm{UI} / 1$. La sideremia era de $43 \mu \mathrm{g} / \mathrm{dl}$ pero la ferritina y la transferrina eran normales. La proteína $\mathrm{C}$ reactiva (PCR) era de 0,50 $\mathrm{mg} / \mathrm{dl}$. Dos coprocultivos mostraron una flora bacteriana saprofita habitual y no se detectó toxina del Clostridium difficile. Con el fin de determinar la causa de la pancreatitis aguda y reconocer la existencia de complicaciones, la enferma fue sometida a las siguientes exploraciones: ecografía abdominal, que fue normal; tomografía axial computerizada (TAC) abdomino-pélvica que mostró una mínima disminución del patrón graso-acinar del páncreas, con captación normal del medio de contraste, sin colecciones líquidas ni aumento de la densidad de la grasa peripancreática. Esta exploración también halló la existencia de numerosas adenopatías mesentéricas de dudoso significado, que no se acompañaban de adenopatías retroperitoneales ni de hepatoesplenomegalia. Se realizó también una colangiorresonancia nuclear que confirmó la presencia de las adenopatías, todas ellas menores de 
$1 \mathrm{~cm}$, pero las vías biliares y pancreáticas eran normales.

A pesar de la buena evolución de la paciente, de encontrarse asintomática y de los resultados de las pruebas de imagen, las tasas en sangre de amilasa y de lipasa permanecieron elevadas (Tabla I).

Tabla I. Gráfica de su evolución a lo largo de las semanas o meses

\begin{tabular}{|c|c|c|c|}
\hline Analítica & Urgencias & 4 días & 26 días \\
\hline $\mathrm{Hb}$ g/dl & 13,6 & 13,2 & 11,5 \\
\hline Hto \% & 40,4 & 38 & 35,1 \\
\hline VCM fl & 91,1 & 90,2 & 94 \\
\hline Plaquetas/mcl & 434.000 & 496.000 & 433.000 \\
\hline Leucocitos/mcl & 7.200 & 4.500 & 4.500 \\
\hline Neutrófilos \% & 79 & 59 & 62 \\
\hline Linfocitos \% & 15,5 & 25 & 24,2 \\
\hline Glucosa mg/dl & 110 & 94 & 78 \\
\hline Creatinina mg/dl & 0,7 & 1,13 & 0,91 \\
\hline Amilasa UI/I & 660 & 624 & 557 \\
\hline Lipasa UI/I & - & 8.859 & 8.910 \\
\hline Prot. totales g/dl & 8,12 & 6,7 & 7,3 \\
\hline Albúmina g/dl & 4,02 & 3,5 & 3,71 \\
\hline Bilirrubina mg/dl & 0,3 & 0,29 & 0,22 \\
\hline GOT UI/I & 31 & 30 & 29 \\
\hline GPT UI/I & 38 & 16 & 39 \\
\hline GGT UI/I & 13 & 12 & 14 \\
\hline F. alcalina UI/I & 171 & 194 & 150 \\
\hline LDH UI/I & 126 & 132 & 128 \\
\hline Ca mg/dl & 8,6 & 8,7 & 8,8 \\
\hline Na mEq/l & 140 & 140 & 142 \\
\hline $\mathrm{K} \mathrm{mEq} / \mathrm{l}$ & 3,8 & 4,67 & 4,48 \\
\hline $\mathrm{Cl}$ mEq/l & 106 & 104 & 107 \\
\hline Coagulación & Normal & Normal & Normal \\
\hline$P C R$ & - & 0,5 & - \\
\hline VSG $1^{\circ}$ hora & & 48 & 60 \\
\hline Hierro $\mu \mathrm{g} / \mathrm{dl}$ & - & 43 & - \\
\hline Ferritina ng/ml & - & 103 & - \\
\hline Transferrina mg/dl & - & 246 & - \\
\hline TIBC $\mu g / d \mid$ & - & 347 & - \\
\hline Dolor abdominal & Intenso & Asintomática & Asintomática \\
\hline Diarrea & No & Intermitente & Intermitente \\
\hline
\end{tabular}

Dra. Yuramí Sánchez. Se trata de una paciente de 34 años, ingresada por presentar una pancreatitis aguda leve que evolucionó favorablemente desde el punto de vista clínico, si bien persistieron elevadas las tasas en sangre de amilasa y lipasa durante, al menos, 26 días. Los estudios radiológicos permitieron descartar la litiasis biliar como causa de la pancreatitis, la cual junto con el consumo excesivo de alcohol constituyen el 75-85\% de las causas de pancreatitis aguda (1). La paciente refería consumo de bebidas alcohólicas muy eventual por causa social y en muy poca cantidad. Entre las causas metabólicas la hipertrigliceridemia es la más frecuente y puede causar pancreatitis en el 1,3 a 3,8\% de los casos (2). La hipercalcemia de cualquier etiología produce pancreatitis aguda de forma muy infrecuente, tanto que su incidencia en los pacientes con hipercalcemia crónica hace pensar que es consecuencia de otros factores, por ejemplo en el caso del hiperparatiroidismo, la pancreatitis aguda se presenta en menos del $1,5 \%$ de los todos los casos $(3,4)$. En esta paciente las tasas normales de triglicéridos y de calcio nos permiten descartar estas causas metabólicas. Se ha descrito que el uso de algunos fármacos (antibióticos, diuréticos, sulfasalazina, agentes inmunosupresores, agentes neuropsiquiátricos, etc.), podría incrementar el riesgo de pancreatitis aguda; sin embargo, esto es bastante infrecuente y la mayoría de las series refieren una incidencia de estos casos del 1-3\% $(3,4)$. También esta etiología se puede descartar en esta paciente, ya que la paciente no consumía habitualmente ningún fármaco. Por ello, con los datos disponibles, la naturaleza de la pancreatitis debemos en principio incluirla en el grupo de las idiopáticas.

Las técnicas de imagen descartaron que existiera cualquier tipo de complicaciones o pseudoquistes que pudieran explicar el aumento persistente de amilasa y lipasa en sangre. Sin embargo, tanto la TAC como la colangiorresonancia nuclear magnética mostraron que existían múltiples adenopatías mesentéricas de tamaño menor de $1 \mathrm{~cm}$ (Fig. 1), cuya significación no se pudo determinar con estas técnicas. En esta paciente, las adenopatías parece que se limitaban al mesenterio, ya que ni la TAC ni la resonancia nuclear magnética pudieron reconocer que existieran en otros lugares anatómicos. Tampoco la exploración física descubrió adenopatías periféricas palpa-

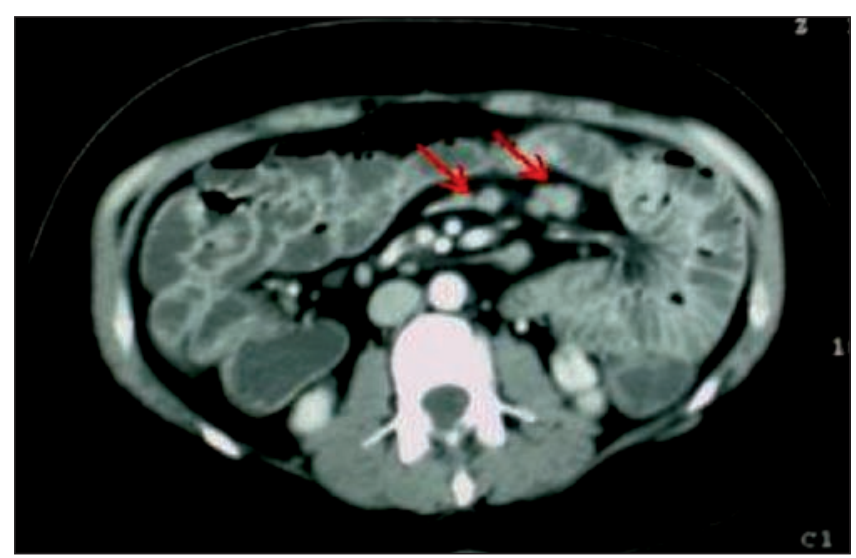

Fig. 1. TAC abdominal en el que se reconocen las adenopatías mesentéricas (flechas).

bles.

En el diagnóstico diferencial de las adenopatías mesentéricas se deben considerar las que aparecen en el curso de enfermedades inflamatorias, infecciosas y tumorales $(5,6)$. Las adenopatías mesentéricas pueden ser secundarias a cualquier proceso inflamatorio, tanto abdominal (apendicitis, diverticulitis, colecistitis, pancreatitis, perforación abdominal) como sistémico. En el caso de la apendicitis, las adenopatías suelen localizarse en el mesenterio de la fosa iliaca derecha. Cuando la causa de las adenopatías es 
una diverticulitis, su localización suele ser en la inmediata vecindad del colon inflamado. Si las adenopatías son secundarias a una pancreatitis, su localización suele ser retroperitoneal o peripancreática. Sólo las formas graves de pancreatitis, que no era el caso de la paciente comentada, pueden acompañarse de adenopatías mesentéricas. En la enfermedad de Crohn, las adenopatías mesentéricas son un hallazgo bastante común $(7,8)$. Estas adenopatías se pueden encontrar tanto en la raíz del mesenterio, como en su periferia o en el cuadrante inferior derecho del abdomen. Es frecuente que se describan como grandes adenopatías, prominentes, aunque es raro que sean masivas. Tras la inyección del contraste radiológico, se produce un aumento de su densidad.

En las colagenosis, incluido el lupus eritematoso, la esclerosis sistémica, la artritis reumatoidea, es frecuente que existan adenopatías, incluso mesentéricas (9-11), pero es muy raro que estas no se acompañen de adenopatías periféricas y retroperitoneales. Otras circunstancias en las que se suelen encontrar adenopatías mesentéricas son la cirrosis biliar primaria (12), la amiloidosis, la mastocitosis, (13) la púrpura de Schönlein-Henoch (14), la fiebre mediterránea familiar (15) y enfermedad celiaca, aunque en todas estas enfermedades, las adenopatías acompañan a las manifestaciones que caracterizan a cada una de ellas. Otra causa inflamatoria de adenopatías mesentéricas es la paniculitis mesentérica. Esta enfermedad también cursa con dolor abdominal, náuseas, vómitos, fiebre y pérdida de peso. Radiológicamente se aprecia que la grasa mesentérica presenta gran atenuación de su densidad originada por la inflamación y la fibrosis $(16,17)$. La exploración con la TAC de la paciente no proporcionó ningún cambio mesentérico que recordara a la que se describe en la paniculitis mesentérica. La sarcoidosis es una enfermedad granulomatosa, multisistémica, de etiología desconocida, relativamente frecuente en adultos menores de 40 años, que afecta preferentemente a los pulmones y al aparato linfoide torácico $(18,19)$, pero que también puede comprometer a los linfáticos abdominales (20). Entre el 30 y el $50 \%$ de los enfermos con sarcoidosis se encuentran asintomáticos y el diagnóstico se alcanza de forma casual al realizar una radiografía de tórax (21). Su diagnóstico es de exclusión y requiere la presencia de: a) manifestaciones clínicas o radiológicas compatibles; b) demostración de granulomas epitelioides no caseificados en las muestras histológicas; y c) la exclusión de otras causas que puedan originar un cuadro clínico-radiológico similar (22). La enferma que comentamos carecía de síntomas respiratorios, tampoco existían síntomas generales, artralgias, eritema nodoso, fiebre, molestias oculares, etc., si bien, como he mencionado más arriba, la enfermedad puede cursar de forma subclínica. Tampoco la radiografía de tórax ni la TAC mostraron cambios anormales. Aunque la biopsia pulmonar transbronquial y el lavado broncoalveolar tienen un gran valor diagnóstico $(18,21,22)$, supongo que en esta enferma no se realizaron al carecer de sintomatología que lo justificara y ser la TAC normal. Aunque creo que la sarcoidosis puede ser excluida en esta enferma como causa de las adenopatías mesentéricas, antes quisiera saber si en esta enferma se determinó la actividad de la enzima convertidora de la angiotensina (ECA), que se encuentra elevada en el 40-80\% de los pacientes con sarcoidosis, en la mayoría de ellos con tasas superiores al doble de su valor máximo normal $(18,19,21)$.

Dra. C. Garfia. Se determinaron los niveles de la ECA los cuales fueron normales $(23,9 \mathrm{U} / 1)$. En efecto, no se realizó biopsia pulmonar ni lavado broncoalveolar.

Dra. Y. Sánchez. Gracias, aunque la normalidad de esta prueba no excluye la sarcoidosis, ya que puede ser normal en el 20-50\% de los enfermos, este dato junto con todos los anteriores creo que nos permiten rechazar esta enfermedad y nos obliga a considerar las opciones infecciosas y tumorales.

Entre las causas infecciosas de adenopatías mesentéricas destaca la tuberculosis. Se trata de una enfermedad que en los últimos años ha vuelto a aumentar en España, probablemente en relación con la llegada de emigrantes procedentes de zonas donde la enfermedad es muy frecuente. Según datos de la OMS, España es el segundo país de la Unión Europea con mayor incidencia de tuberculosis (después de Portugal), con más de 20 casos/100.000 habitantes (23). Las adenopatías tuberculosas suelen tener características particulares, ya que cuando la tuberculosis pulmonar es primaria, las adenopatías suele ser unilaterales y los ganglios afectados son los paratraqueales y subclaviculares; cuando la tuberculosis es miliar, el patrón radiológico es uniforme y difuso, con adenopatías de entre 10 y $30 \mathrm{~mm}$, en ocasiones formando conglomerados o calcificadas $(24,25)$. Las adenopatías abdominales se localizan preferentemente en el mesenterio en lugar de en el retroperitoneo. En el $60 \%$ de los casos muestran una atenuación central ocasionada por la necrosis central (26). Tras la inyección intravenosa de contraste, se produce un refuerzo periférico de su imagen (25). Un aspecto radiológico similar, cavitado, se ha descrito en la enfermedad de Whipple causada por la Tropheryma whippelii (27) y en la enfermedad celiaca $(28,29)$. Cuando la tuberculosis es primaria intestinal, las adenopatías pueden limitarse al mesenterio. La enferma que comentamos carecía de sintomatología respiratoria y las adenopatías no presentaban las características radiológicas de la tuberculosis, por lo que esta etiología parece sumamente improbable; no obstante, antes de descartar que se tratara de una tuberculosis nos gustaría conocer si se hizo el estudio microbiológico del esputo, prueba de Mantoux, radiografías póstero-anteriores y lateral de tórax y, a ser posible, TAC de tórax.

Dra. C. Garfia. El esputo no se pudo analizar ya que carecía de sintomatología respiratoria y de expectoración. La radiografía de tórax fue informada por el radiólogo como normal y la TAC de tórax con doble contraste no mostró alteraciones parenquimatosas ni adenopatías mediastínicas. La prueba del Mantoux fue negativa. 
Dra.Y. Sánchez. Bien, siendo improbable la etiología tuberculosa de las adenopatías mesentéricas, debemos considerar otras causas infecciosas. La infección por Yersinia enterocolitica es una de ellas. Es característico de esta infección el engrosamiento de la pared del íleon terminal acompañado de adenopatías mesentéricas (30). La sintomatología puede confundirse con la provocada por la apendicitis aguda y las lesiones intestinales con la enfermedad de Crohn. También la infección por el virus de la inmunodeficiencia humana $(\mathrm{VIH})$ puede originar linfoadenopatías mesentéricas en el 10-15\% de los casos. En unas ocasiones, ello se debe a la propia infección viral, en otras son originadas por otras infecciones oportunistas o por complicaciones neoplásicas (31). En el primer caso, el tamaño de las adenopatías es pequeño o mediano, pero no masivo. Entre las infeccionas oportunistas más frecuentes en estos pacientes es la provocada por el complejo del Mycobacterium avium; esto ocurre cuando la tasa de CD4 es menor de 50/ml. En el $42 \%$ de los enfermos con esta infección sobreañadida se encuentran adenopatías mesentéricas, habitualmente masivas y formando masas conglomeradas $(30,32)$. Las características de las adenopatías mesentéricas de la enferma que comentamos eran muy diferentes a las originadas por el $M y$ cobacterium avium. La adenitis mesentérica es otra causa común de adenopatías mesentéricas preferentemente en la infancia, si bien, las técnicas de imagen nos están demostrando que tampoco son raras en los adultos (26). Se trata de una inflamación benigna de etiología incierta, habitualmente viral $(33,34)$, en la que existen adenopatías mesentéricas, en ocasiones acompañadas de inflamación intestinal. Estos enfermos suelen aquejar dolor abdominal agudo, crónico o recurrente, localizado preferentemente en la fosa iliaca derecha, frecuentemente acompañado de náuseas, vómitos, diarrea, fiebre y leucocitosis, por lo que no es raro que sea confundido con apendicitis aguda $(26,35)$. Desconozco si se realizaron estudios para descartar las infecciones abdominales que he mencionado. Si no es así, estas enfermedades no deben ser olvidadas (Tabla II).

Tabla II. Enfermedades causantes de adenopatías mesentéricas

\begin{tabular}{lll}
\hline Infecciosas & Inmunológicas & Hematológicas \\
\hline Adenitis mesentérica & Lupus eritematoso & Linfoma Hodgkin \\
Tuberculosis & Artritis reumatoide & Linfoma no Hodgkin \\
Yersinia enterocolitica & Dermatomiositis & Leucemia linfoide crónica \\
Y. pseudotuberculosis & Sarcoidosis & Linfoma linfoblástico de células T \\
Micosis & $\begin{array}{l}\text { Paniculitis mesentérica } \\
\text { Leucemia aguda linfoblástica }\end{array}$ & $\begin{array}{c}\text { de células T } \\
\text { Enfermedad de Crohn }\end{array}$ \\
Enfermedad de Whipple & Cirrosis biliar primaria & Metástasis \\
Mononucloosidosis & \\
Citomegalovirus & Mastocitosis & \\
Rubéola & Púrpura de Schönlein-Henoch \\
Sífilis & Enfermedad celiaca & \\
Toxoplasmosis & & \\
Micosis & & \\
Sida & & \\
\hline
\end{tabular}

El tercer grupo de adenopatías mesentéricas que debo considerar es el de las tumorales, principalmente el de las provocadas por los procesos linfoproliferativos (36). La enfermedad de Hodgkin suele iniciarse en los ganglios linfáticos del mediastino o del cuello y desde ellos se extiende a otras áreas ganglionares, incluido el retroperitoneo y el bazo. Más adelante, la enfermedad se disemina por vía hematógena y compromete a diversos órganos, tales como el hígado, pulmón o médula ósea. La enferma comentada carecía de adenopatías supradiafragmáticas, síntomas B (fiebre, sudoración nocturna, pérdida de peso) o signos de afectación hepática o esplénica. Aunque más raro, la enfermedad puede presentarse con afectación infradiafragmática exclusiva en el 30\% de los casos, si bien, en estos casos, las adenopatías son retroperitoneales y no mesentéricas $(25,37)$. El diagnóstico definitivo de enfermedad de Hodgkin se realiza cuando se demuestra la presencia de células de Reed-Sternberg en el estudio histológico de las adenopatías (37). Como supongo que las adenopatías mesentéricas de esta paciente no fueron biopsiadas, desearía saber si en ella se realizó una gammagrafía abdominal con $67 \mathrm{Ga}$ o una tomografía por emisión de positrones (PET) utilizando 18F-doxiglucosa. Ambas exploraciones son muy útiles para reconocer el carácter linfomatosos de las adenopatías (38).

Dra. C. Garfia. En esta enferma se realizó una gammagrafía abdominal con $67 \mathrm{Ga}$, que no mostró cambios que sugirieran la existencia de un linfoma.

Dra. Y. Sánchez. Con estos resultados es aún más improbable que las adenopatías mesentéricas de esta enferma se deban tanto a un linfoma Hodgkin como al no Hodgkin. No obstante, en estos últimos y, en contra de lo que ocurre en la enfermedad de Hodgkin, las primeras manifestaciones pueden ser extraganglionares, incluidas las derivadas de la afectación del tracto gastrointestinal y los ganglios linfáticos del mesenterio (37). Inicialmente, las adenopatías son escasas y pequeñas, pero a medida que la enfermedad avanza, su tamaño y número aumenta y tienden a confluir formando grandes masas de tejido blando que desplazan a las estructuras vecinas. En la TAC, su densidad es la propia de los tejidos blandos, pero en ocasiones existe un anillo periférico de mayor densidad. Tampoco estos cambios radiográficos son los que se hallaron en la enferma comentada.

Otros tumores que frecuentemente originan adenopatías mesentéricas son los carcinomas de mama (39), pulmón, páncreas, gastrointestinales $(40,41)$ y vesícula, el melanoma y los sarcomas, incluido el de Kaposi (42). Los tumores de mama y pulmón rara vez provocan adenopatías mesentéricas sin antes afectar al mediastino, hilio pulmonar o axilas. Los gastrointestinales y de colon se asocian muy frecuentemente con adenopatías mesentéricas. El carcinoma de páncreas origina también adenopatías de esa localización; sin embargo, las primeras adenopatías en aparecer son las peripancreáticas y retroperitoneales. El sarcoma de Kaposi origina grandes 
adenopatías, de 3 a $5 \mathrm{~cm}$, que tienden a confluir formando grandes masas y suelen acompañarse de compromiso intestinal. La presencia de adenopatías localizadas en un solo territorio obliga a explorar los órganos vecinos, ya que pueden ser la única manifestación de una enfermedad neoplásica $(24,43,44)$.

En el caso de esta paciente con adenopatías exclusivamente mesentéricas es obligado el estudio del tubo digestivo. Tumores de esta localización, en especial los linfomas, pueden originar este tipo de adenopatías mesentéricas (45). Los tipos de linfomas que más frecuentemente afectan al aparato digestivo son: a) linfomas $B$ de la zona marginal del tejido linfático asociado a las mисоsas (linfomas MALT); b) linfomas $B$ difuso de células grandes (linfoma MALT de alto grado); y c) otros linfomas (linfoplasmocítico; de células del manto; de Burkitt; de células $T$ asociados o no a enteropatía). Los linfomas MALT se suelen manifestar con diversos cuadros clínicos entre los que figura la diarrea, la malabsorción intestinal, el dolor abdominal, los síntomas generales o de obstrucción intestinal. Estos tumores, que inicialmente se limitan a la pared del tubo digestivo, se extienden hacia los ganglios mesentéricos y, más tarde, a grupos ganglionares más alejados: aproximadamente el 13\% de los casos presentan adenopatías subdiafragmáticas en el momento del diagnóstico (46). Los linfomas T intestinales son más raros, pero en muchos casos se desarrollan en pacientes con enfermedad celiaca (47). Por esta razón, desearía saber si se hicieron estudios radiológicos o endoscópicos del tubo digestivo. Estos estudios, además, nos ayudarían a descartar o a apoyar algunos de los diagnósticos comentados (tu- berculosis, yersiniosis, enfermedad inflamatoria intestinal, enfermedad celiaca, etc.).

Dra. C. Garfia. Sí se hicieron. El estudio radiológico del tránsito intestinal mostró que las asas intestinales estaban algo dilatadas, pero su diámetro se encontraba aún dentro de los límites de la normalidad. Únicamente, en el íleon superaban estos límites. Sin embargo, las características de los pliegues eran normales (Fig. 2). También se realizó una colonoscopia, que no descubrió ningún cambio anormal. Una gastroscopia reveló la existencia de una imagen endoscópica de gastritis crónica de predominio antral y una atrofia vellositaria duodenal. Por esta razón, se realizó también el estudio del intestino delgado con cápsula endoscópica, el cual confirmó que existía una atrofia vellositaria leve de duodeno y yeyuno proximal, pero no masas, zonas infiltradas, úlceras o estenosis (Fig. 3).

Dra. Y. Sánchez. Evidentemente, estos estudios sugieren que la enferma pudiera padecer una enfermedad intestinal que curse con atrofia vellositaria. Aunque esta atrofia forma parte del espectro de lesiones histológicas que ocurren en la enfermedad celiaca, no es la única enfermedad en la que existe atrofia de las vellosidades intestinales. En efecto, este cambio se puede encontrar en las parasitosis, sobrecrecimiento bacteriano, enteritis eosinofílica, esprúe tropical, malnutrición, hipogammaglobulinemia, gastroenteritis aguda viral, intolerancia a la leche de vaca, entre otras. Para una correcta interpretación de los resultados histológicos es necesario disponer de más datos histológicos, que incluyan el estado de las criptas glandulares, las características del epitelio y el

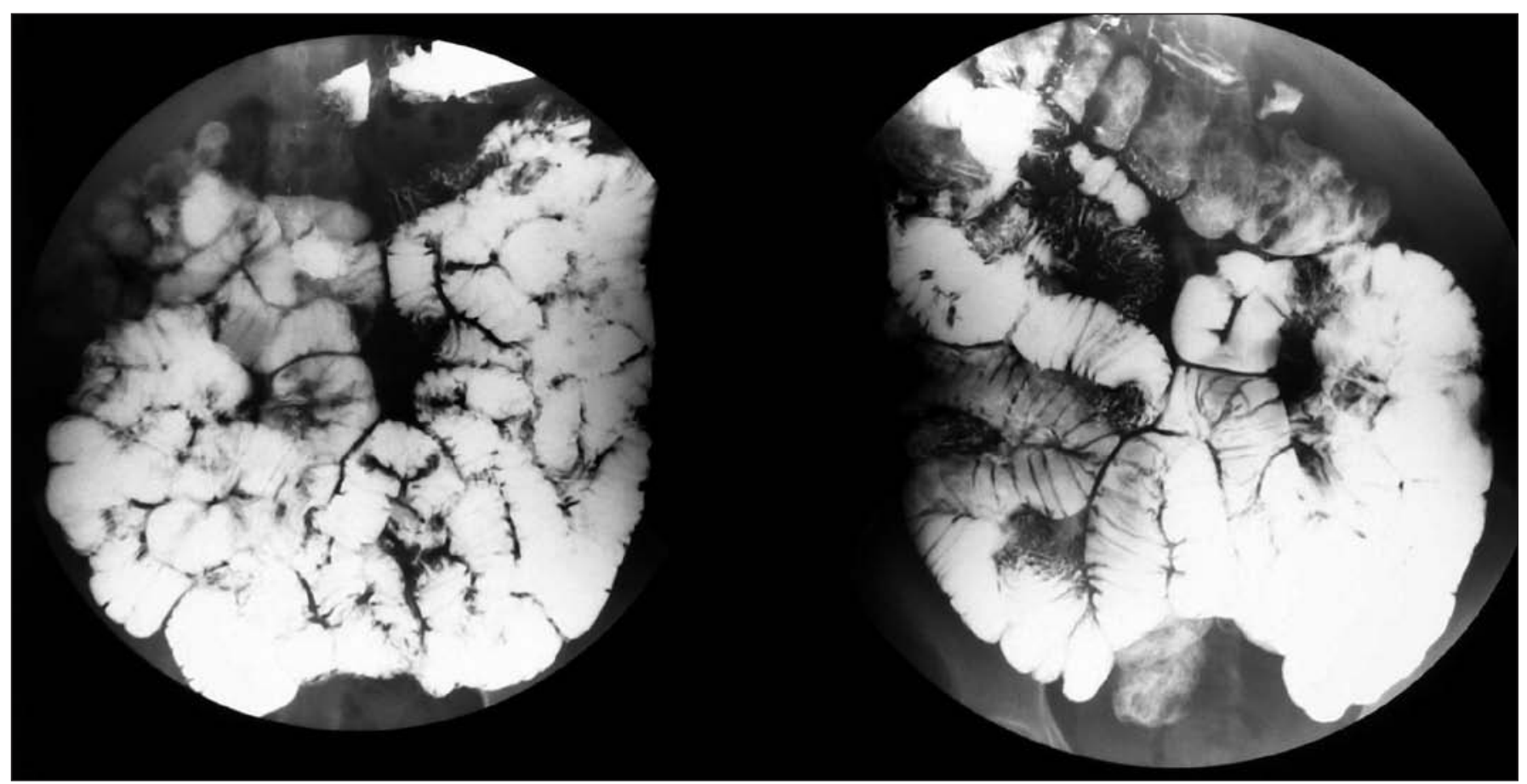

Fig. 2. Tránsito intestinal. Obsérvese el ligero aumento del calibre de las asas y del grosor de los pliegues intestinales 


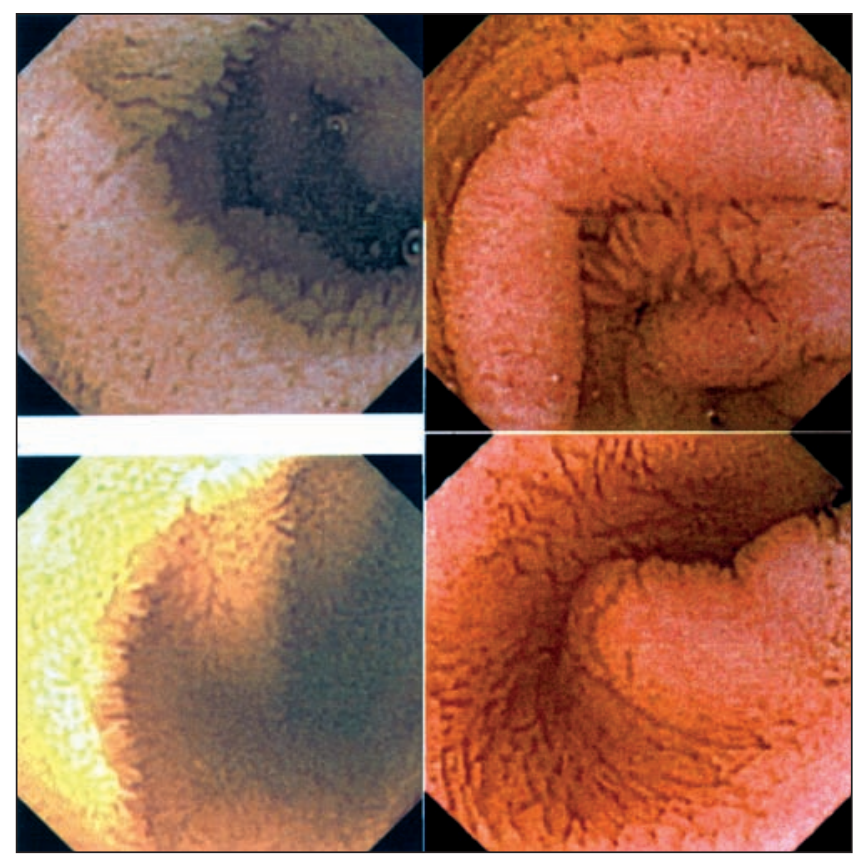

Fig. 3. Imágenes obtenidas mediante la cápsula endoscópica que muestran atrofia vellositaria leve en duodeno y yeyuno proximal.

tipo de infiltrado inflamatorio. En la enfermedad celiaca existe un acortamiento o aplanamiento de las vellosidades intestinales a la vez que una hiperplasia de las criptas glandulares y una infiltración de la lámina propia principalmente por linfocitos (CD4+) y células plasmáticas $(47,48)$. Estas lesiones, determinadas por la exposición a los alimentos que contienen gluten, es frecuente entre los europeos y sus descendientes en otros países, en los que puede llegar a presentarse en 1 por cada 100-300 habitantes (49-51). El riesgo de padecer esta enfermedad es especialmente alto entre los familiares de primer y segundo grado de pacientes con enfermedad celiaca, en los pacientes con diabetes mellitus de tipo I, en los que presentan tiroiditis u otras enfermedades autoinmunes (52) y en pacientes con enfermedad de Down como ocurre en la enferma que comentamos. En esta última situación su prevalencia es del 3 al $12 \%$ y se estima que el riesgo de estos enfermos para padecer una enfermedad celiaca es unas cinco veces superior al de la población general $(51,53)$. Por ser en nuestro medio la enfermedad celiaca relativamente frecuente (54) y por cursar en los adultos en la mayoría de las ocasiones de forma subclínica, oligosintomática o con manifestación no digestiva, es por lo que empezaré por analizar la posibilidad de que esta enferma padezca esta enfermedad. Para ello necesito saber los resultados de la determinación de las grasas en heces, de los anticuerpos anti-transglutaminasa y de la biopsia intestinal.

Dra. C. Garfia. Se midieron las grasas en heces, pero estas fueron normales ( $1 \mathrm{~g} / 24$ horas), así como los niveles de quimotripsina fecal $(27,1 \mathrm{U} / \mathrm{g})$; las tasas en sangre tras la administración oral de $25 \mathrm{~g}$ de D-Xilosa fueron de
$24,7 \mathrm{mg} \%$ y $31 \mathrm{mg} \%$ a los 60 y $120 \mathrm{~min}$, respectivamente, y su eliminación urinaria a las 5 horas de $14 \%$ (3,5 g), todos ellos dentro de la normalidad. Los niveles de inmunoglobulinas en sangre fueron normales (IgG, 1270 $\mathrm{mg} / \mathrm{dl}, \mathrm{IgA}, 484 \mathrm{mg} / \mathrm{dl}, \mathrm{IgM}, 62 \mathrm{mg} / \mathrm{dl})$ y los anticuerpos no órganos específicos (ANA, AML, AMA, ALKM) fueron negativos. Por el contrario, los anticuerpos antigliadina-IgA fueron de 45,6 UI/1 y los antitransglutaminasaIgA de 581,7 UI/1 (ambos elevados). En relación con los hallazgos histológicos de las biopsias tomadas durante la gastroscopia, fueron descritos como: biopsia de mucosa duodenal que muestra una alteración arquitectural constituida por atrofia total de las vellosidades, hiperplasia de las criptas glandulares e infiltrado linfoplasmocitario en la lámina propia (Fig. 4). La biopsia de mucosa gástrica (antral, fúndica) mostraba una población glandular normal, con escaso infiltrado de neutrófilos, sin infiltrado linfoide en folículos, ni bacilos reconocibles con la tinción de Giemsa. Estos estudios histológicos concluyeron que existía una mucosa duodenal con atrofia total de vellosidades y una gastritis superficial antral, sin evidencia de Helicobacter pylori.

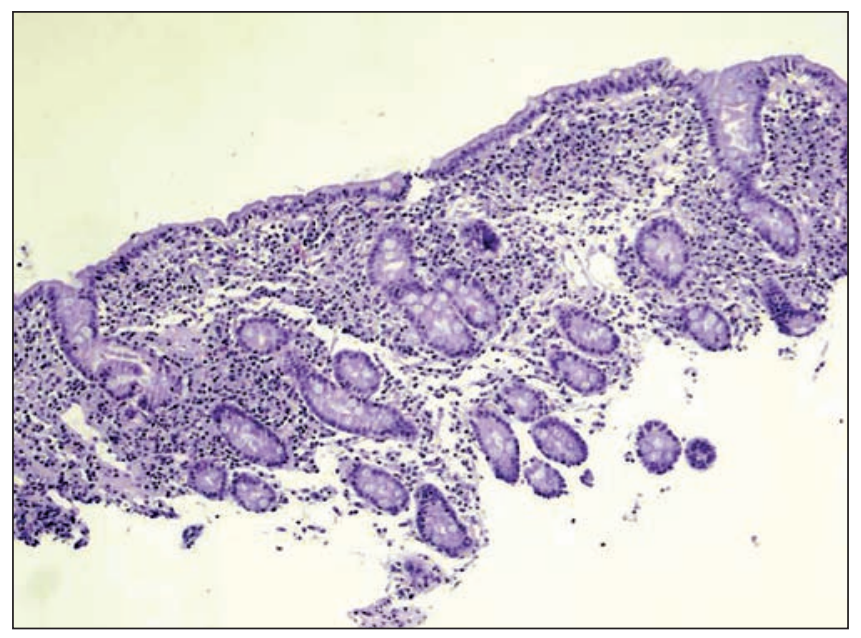

Fig. 4. Biopsia de mucosa duodenal distal que muestra atrofia total de las vellosidades intestinales, hiperplasia de las criptas glandulares e infiltración de la lámina propia.

Dra. Y. Sánchez. Todos estos hallazgos sugieren que la enferma pudiera padecer una enfermedad celiaca del adulto hasta entonces poco sintomática, pero que justificaría los episodios de diarrea que figuran en la historia clínica, así como las molestias epigástricas. La normalidad de las grasas en heces se pudiera explicar, aún existiendo una enfermedad celiaca, si la recogida de las heces no fue correcta o la ingesta de grasa fue insuficiente. No obstante, existen casos en los que la absorción intestinal de grasas es normal pero está defectuosa la absorción de otros nutrientes. En cualquier caso, una excreción fecal normal de grasa no excluye una enfermedad celiaca. La 
prueba de la D-xilosa aportó resultados que se encontraban en los límites bajos de la normalidad, ya que su excreción urinaria debe ser superior a los $4 \mathrm{~g}(>16 \%)$ y su concentración en sangre a los 60 minutos superar los 20 $\mathrm{mg} / \mathrm{dl}$. Tampoco la fiabilidad diagnóstica de esta prueba es demasiado alta, ya que puede ser normal hasta en el $20 \%$ de los pacientes con enfermedad celiaca no tratada (55). La sensibilidad y especificidad de los anticuerpos antitransglutaminasa-IgA en el diagnóstico de la enfermedad celiaca son muy altas, superiores al $95 \%$ cuando existe atrofia vellositaria total (56), como era el caso de la paciente. Por esta razón, está justificado retirar de la dieta los alimentos con gluten y observar la evolución de la paciente. El diagnóstico de enfermedad celiaca se reforzaría si la sintomatología abdominal, las tasas de anticuerpos transglutaminasa y las lesiones histológicas mejoraran en pocos días o semanas tras retirar totalmente el gluten de la dieta $(51,57)$. La normalización histológica, sin embargo, suele requerir habitualmente más de un año o es posible que no se produzca nunca $(58,59)$, si bien, la altura de las células epiteliales del muñón de las vellosidades aumenta durante las primeras semanas que siguen a la retirada del gluten de la dieta. La TAC y la resonancia nuclear magnética pueden proporcionar datos que tienen gran valor diagnóstico, por ejemplo, la atrofia esplénica, la ascitis y las linfoadenopatías (60-62). Estas últimas se encuentran en el $43 \%$ de los casos (63) y son sumamente sugerentes de esta enfermedad cuando aparecen cavitadas $(28,29,64)$, si bien se trata de un hallazgo raro en esta enfermedad, descrito en menos de 50 ocasiones. Cuando se estudian histológicamente, se encuentra que las adenopatías están cavitadas, ocupadas por un material quilosocremoso y rodeadas por un anillo periférico delgado compuesto por fibrosis y restos del ganglio atrofiado (65). Su hallazgo permite reconocer que la causa de las adenopatías no es un linfoma complicando a la enfermedad celiaca. La relación de las adenopatías mesentéricas con la enfermedad celiaca se demuestra cuando su número y tamaño disminuye al retirar el gluten de la dieta (62). Por todo ello, desearía conocer si a esta enferma se le retiró el gluten de la dieta y, en este caso, cuál fue la respuesta.

Dra. C. Garfia. Con los datos de que disponíamos, también nosotros pensamos en la enfermedad celiaca y, en consecuencia, le retiramos de su dieta los alimentos que contienen gluten. La evolución de la paciente fue la de una completa desaparición del dolor y de las molestias digestivas y de un descenso de las tasas en sangre de amilasa y de lipasa, que a los 6 meses habían descendido a $266 \mathrm{UI} / 1$ y $1.724 \mathrm{UI} / 1$, respectivamente. Un curso similar han seguido los anticuerpos antigliadina- $\operatorname{IgA}(21,8$ $\mathrm{UI} / \mathrm{ml})$ y antitransglutaminasa-IgA $(368,5 \mathrm{UI} / \mathrm{ml})$.

Dra. Y. Sánchez. La respuesta de esta paciente a la deprivación del gluten apoya que la enfermedad que padece sea una celiaca, si bien, a los seis meses de supresión del gluten, las tasas de esos anticuerpos deberían ser aún más bajas. En general, cuando la restricción del gluten de la dieta es completa, las tasas en sangre de esos anticuerpos vuelven a lo normal en el plazo de 3 a 6 meses $(59,60)$. Por ello, es muy probable que la enferma no estuviera haciendo la dieta correctamente y que siguiera ingiriendo alimentos con gluten aunque fuera de forma involuntaria (por olvido o descuido) como sucede en muchos pacientes no respondedores a la dieta (66). Durante la restricción dietética, las tasas en sangre de amilasa y de lipasa descendieron, lo que sugiere que la enfermedad pancreática también estaba relacionada con la enfermedad celiaca. Hay varios estudios que refieren la existencia de hiperamilasemia e hiperlipasemia en la enfermedad celiaca y su mejoría progresiva con la dieta (67). En el caso comentado, hubo descenso pero no normalización de las enzimas pancreáticas en sangre. La regresión incompleta de estos cambios pudiera estar también en relación con el mal cumplimiento de la restricción dietética; sin embargo, en ocasiones, a pesar de que la dieta se siga estrictamente, el descenso puede ser muy lento y requerir hasta uno o dos años para su normalización (67). Estas elevaciones de las enzimas pancreáticas pudieran justificarse por la existencia de una pancreatitis autoinmune asociada a la enfermedad celiaca (68). Aproximadamente, el 20\% de los pacientes con celiaca presentan alguna enfermedad autoinmune asociada $(52,69)$, las más frecuentes son la diabetes mellitus del tipo I, el hipotiroidismo, el lupus eritematoso y la gastritis atrófica. No existen criterios claros para el diagnóstico de pancreatitis autoinmune, pero es frecuente que los conductos pancreáticos presenten un calibre irregular, estrecho y que las tasas en sangre de IgG, en especial de la IgG4, se encuentren muy elevadas $(70,71)$. La enferma que comentamos no mostró cambios importantes en los conductos pancreáticos apreciables por colangiorresonancia y la electroforesis no mostró que existiera un aumento llamativo de la IgG. Aunque el valor diagnóstico de esta última prueba ha sido cuestionada, en la enfermedad celiaca se han hallado otras alteraciones que pudieran justificar las alteraciones pancreáticas (69). Por ejemplo: macroamilasemia con o sin macrolipasemia (72-74), la inflamación duodenal, la estenosis de la papila de Vater o la escasa producción de pancreozimina en la enfermedad celiaca pudieran ser responsables de esas alteraciones $(73,75,76)$.

Aunque en el desarrollo de las lesiones intestinales de la enfermedad celiaca juega un papel decisivo la intolerancia al gluten, existe sin duda una fuerte predisposición genética $(47,77)$. En este sentido se ha encontrado que el 80-95\% de los enfermos con celiaca poseen el HLA-DQ2 $(\alpha 1 * 501, \beta 1 * 02)$ y el HLA-DQ8 $(\alpha 1 * 0301, \beta 1 * 0302)$ en los restantes $(47,53,78,79)$. Por ello, desearía conocer si en esta enferma se realizó este estudio genético.

Dra. C. Garfia. Sí se realizó y se halló que la enferma es homocigoto para el HLA DQ2.

Dra. Y. Sánchez. Es decir, este estudio genético también apoya el diagnóstico de enfermedad celiaca. Aunque este HLA-DQ2 es bastante común en la población europea $(25-30 \%)$ sin sufrir la enfermedad celiaca, se 
sabe que cuando el HLA DQ2 aparece en ambos cromosomas, el riesgo de padecer una enfermedad celiaca se multiplica por cinco en relación con el riesgo existente en los heterocigotos $(79,80)$.

Aunque como hemos dicho antes, en la enfermedad celiaca se pueden encontrar adenopatías mesentéricas, se trata de un hallazgo raro y suelen ir desapareciendo con la restricción dietética, por ello nos gustaría conocer la evolución de las mismas tras la retirada del gluten de la dieta.

Dra C. Garfia. A los 6 meses de instaurar la dieta libre de gluten se realizó una nueva TAC, que demostró la persistencia de las adenopatías descritas. Una de ellas era de 1,7 x $0,9 \mathrm{~cm}$ y otra de $1,9 \times 1,2 \mathrm{~cm}$ que parecían corresponder más que adenopatías únicas a conglomerados de otras adenopatías más pequeñas, pero seguía sin visualizarse adenopatías en otros territorios.

Dra Y. Sánchez. Este hallazgo obliga a mantener una vigilancia estrecha de esta paciente, con controles tomográficos periódicos.

Dra C. Garfia. Efectivamente y aunque en ninguna de las pruebas diagnósticas realizadas se habían encontrado evidencias de otros procesos, fundamentalmente tuberculosis o linfoma, dada la persistencia de las adenopatías mesentéricas, se realizó una laparoscopia diagnóstica con biopsia de las adenopatías mesentéricas. En esta se observaron adenopatías de pequeño tamaño y de características histológicas inespecíficas.

\section{DIAGNÓSTICO FINAL}

Enfermedad celiaca del adulto, pancreatitis aguda y adenopatías mesentéricas inespecíficas.

\section{BIBLIOGRAFÍA}

1. Whitcomb D. Acute pancreatitis. New Eng J Med 2006; 354: 214-50.

2. Fortson MR, Freedman SN, Webster PD 3rd. Clinical assessment of hyperlipidemic pancreatitis. Am J Gastroenterol 1995; 90: 2134-9.

3. Ward JB, Petersen OH, Jenkins SA, Sutton R. Is an elevated concentration of acinar cytosolic free ionised calcium the trigger for acute pancreatitis? Lancet 1995; 346: 1016-9.

4. Yamada T, Hasler W, Inadomi J, Anderson M, Brown R. Handbook of Gastroenterology. $2^{\mathrm{a}}$ ed. Philadelphia: Lippincottt Williams \& Wilkins; 2005.

5. Rathaus V, Shapiro M, Grunebaum M, Zissin R. Enlarged mesenteric lymph nodes in asymptomatic children: the value of the finding in various imaging modalities. Br J Radiol 2005; 78: 30-3.

6. Lucey BC, Stuhlfaut JW, Soto JA. Mesenteric lymph nodes seen at imaging: causes and significance RadioGraphics 2005; 25: 351-65.

7. Goldberg HI, Gore RM, Margulis AR, Moss AA, Baker EL. Computed tomography in the evaluation of Crohn disease. AJR Am J Roentgenol 1983; 140: 277-82.

8. Nagamata H, Inadama E, Arihiro S, Matsuoka M, Torii A, Fukuda K The usefulness of MDCT in Crohn's disease. Nippon Shokakibyo Gakkai Zasshi 2002; 99: 1317-25.

9. Calguneri M, Ozturk MA, Ozbalkan Z, Akdogan A, Ureten K, Kiraz $\mathrm{S}$, et al. Frequency of lymphadenopathy in rheumatoid arthritis and systemic lupus erythematosus. J Int Med Res 2003; 31: 345-9.
10. Radin R. Acute mesenteric and retroperitoneal lymphadenitis in systemic lupus erythematosus: case report. Abdom Imaging 2001; 26: 411-3.

11. Kitsanou M, Andreopoulou E, Bai MK, Elisaf M, Drosos AA. Extensive lymphadenopathy as the first clinical manifestation in systemic lupus erythematosus. Lupus 2000; 9: 140-3.

12. Outwater E, Kaplan MM, Bankoff MS. Lymphadenopathy in primary biliary cirrhosis: CT observations. Radiology 1989; 171: 731-3.

13. Ben Romdhane K, Ben Romdhane N, Ben Younes MR, Ayadi S, Ben Ayed M. Systemic mastocytosis: a case report. Arch Anat Cytol Pathol 1990; 38: 100-3.

14. Jeong YK, Ha HK, Yoon CH, Gong G, Kim PN, Lee MG, et al. Gastrointestinal involvement in Henoch-Schönlein syndrome: CT findings. AJR Am J Roentgenol 1997; 168: 965-8.

15. Zissin R, Rathaus V, Gayer G, Shapiro-Feinberg M, Hertz M. CT findings in patients with familial Mediterranean fever during an acute abdominal attack. Br J Radiol 2003; 76: 22-5.

16. Horton KM, Lawler LP, Fishman EK. CT findings in sclerosing mesenteritis (panniculitis): spectrum of disease. RadioGraphics 2003; 23: $1561-7$.

17. Valls C. Fat-ring sign in sclerosing mesenteritis. AJR Am J Roentgenol 2000; 174: 259-60.

18. American Thoracic Society. Statement on sarcoidosis. Am J Respir Care Med 1999; 160: 136-55.

19. Wu J, R K. Sarcoidosis. Am Fam Phy 2004; 70(2): 312-22.

20. Fazzi P, Solfanelli S, Morelli G, Orsitto E, Pieri L, Petrini M, et al. Sarcoidosis: single bulky mesenteric lymph node mimicking a lymphoma. Sarcoidosis 1995; 12: 75-7.

21. Lanuzzi M, Rybicki B, Teirstein A. Sarcoidosis. New Engl J Med 2007; 357: 2153-65.

22. Baughman R, Iannuzzi M. Diagnosis of sarcoidosis: when is a peek good enough? Chest 2000; 117: 931-2

23. Balagué M, Orcau A, Sánchez P, Torjada C, Caylà JA. Epidemiología actual de la tuberculosis en España: hacia una mejor vigilancia y control. Programa de prevención y control de la TBC y Unidad de Investigación en TBC de Barcelona. Servicio de epidemiología. Agencia de Salud Pública de Barcelona. Disponible en: http://www.seimc.org/control/revi_Micobac/pdf/Epitbc.pdf.

24. Andreu J, Cáceres J, Pallisa E, Martínez-Rodríguez M. Radiological manifestations of pulmonary tuberculosis. Eur J Radiol 2004; 51: 139-49.

25. Yang ZG, Min PQ, Sone S, He ZY, Liao ZY, Zhou XP, et al. Tuberculosis versus lymphomas in the abdominal lymph nodes: evaluation with contrast-enhanced CT. Am J Roentgenol 1999; 172: 619-23.

26. Zarewych ZM, Donnelly LF, Frush DP, Bisset GS. Imaging of pediatric mesenterio abnormalities. Pediatr Radiol 1999; 29: 711-9.

27. Friedman HD, Hadfield TL, Lamy Y, Fritzinger D, Bonaventura M, Cynamon MT. Whipple's disease presenting as chronic wastage and abdominal lymphadenopathy. Diagn Microbiol Infect Dis 1995; 23: 111-3.

28. Schmitz F, Herzig KH, Stuber E, Tiemann M, Reinecke-Lüthge A, Nitsche R, et al. On the pathogenesis and clinical course of mesenteric lymph node cavitation and hyposplenism in coeliac disease. Int $\mathrm{J}$ Colorectal Dis 2002; 17:192-8.

29. Al-Kawas FH, Murgo A, Foshag L, Shiels W. Lymphadenopathy in celiac disease: not always a sign of lymphoma. Am J Gastroenterol 1988; 83: 301-3.

30. Trommer G, Bewer A, Kosling S. Mesenteric lymphadenopathy in Yersinia enterocolitica infection. Radiologe 1998; 38: 37-40.

31. Radin R. HIV infection: analysis in 259 consecutive patients with abnormal abdominal CT findings. Radiology 1995; 197: 712-22.

32. Pantongrag-Brown L, Krebs TL, Daly BD, Wong-You-Cheong JJ, Beiser C, Krause B, et al. Frequency of abdominal CT findings in AIDS patients with M. avium complex bacteraemia. Clin Radiol 1998; 53: 816-9.

33. Rao PM, Rhea JT, Novelline RA. CT diagnosis of mesenteric adenitis. Radiology 1997; 202: 145-9.

34. Macari M, Hines J, Balthazar E, Megibow A. Mesenteric adenitis: CT diagnosis of primary versus secondary causes, incidence, and clinical significance in pediatric and adult patients. AJR Am J Roentgenol 2002; 178: 853-8.

35. Karmaza B, Werner EA, Rejaie B, Applegate KE. Mesenterio lymh nodes in children: What is normal? Pediatric Radiol 2005; 35: 774-7.

36. Cole SM. Computed tomography of lymphomatous involvement of 
mesenteric nodes. Radiography 1986; 52: 299-301.

37. Kumar V, Robbins SL, Cotran RS. Patología Humana. $7^{\mathrm{a}}$ ed. España: Elsevier; 2005

38. Begerter M, Moog F, Griesshammer M, Elsner K, Kotzerke J, Heimpel $\mathrm{H}$, et al. Role of whole body FDG-PET imaging in predicting relapse of malignant lymphoma in patients with residual masses after treatment. Radiography 1999; 5: 155-63.

39. Hansen RM, Lewis JD, Janjan NA, Komorowski RA. Occult carcinoma of the breast masquerading as primary adenocarcinoma of the small intestine: a case report. J Clin Gastroenterol 1988; 10: 213-7.

40. Shirkhoda A, Staab EV, Bunce LA, Herbst CA, McCartney WH. Computed tomography in recurrent or metastatic colon cancer: relation to rising serum carcinoembryonic antigen. J Comput Assist Tomogr 1984; 8: 704-8.

41. Chintapalli KN, Chopra S, Ghiatas AA, Esola CC, Fields SF, Dodd GD 3rd. Diverticulitis versus colon cancer: differentiation with helical CT findings. Radiology 1999; 210: 429-35.

42. Moon KL Jr, Federle MP, Abrams DI, Volberding P, Lewis BJ. Kaposi sarcoma and lymphadenopathy syndrome: limitations of abdominal CT in acquired immunodeficiency syndrome. Radiology 1984; 150: 479-83.

43. Pzarro S. Adenopatías localizadas y generalizadas. En: Guía de actuación en atención primaria. Barcelona: SEMFYC; 1998. p. 19-22.

44. Dugosory G, Ergogar N, Gulman Y, Gülmen M, Tüzüner N, Ulkü B. Inflammatory pseudotumor of pelvic lymph nodes. Arch Anat Cytol Pathol 1996; 44: 112-24.

45. Feldman M, Friedman L, Sleinseger M. Enfermedades Gastrointestinales y Hepáticas. Fisiopatología, diagnóstico y tratamiento. $7^{\mathrm{a}} \mathrm{ed}$ España: Panamericana; 2002.

46. Gisbert JP, Aguado B, Luna M, Nistal S, Asenjo LM, Reina T, et al Gastric MALT lymphoma: clinical chharacteristics and prevalence of H. pylori infection in a series of 37 cases. Rev Esp Enferm Dig 2006; 98(9): 655-65.

47. Green P, Cellier Ch. Celiac Disease. New Eng J Med 2007; 357: $1731-43$

48. Mino-Kenudson M, Brown I, Lauwers GY. Histopathological diagnosis of gluten sensitive enteropathy. Current Diagnostic Pathology 2005; $11: 274-83$

49. Green P, Jabri B. Coeliac disease. The Lancet 2003; 362: 383-91.

50. Farrel RJ, Kelly CP. Celiac sprue. N Eng J Med 2002; 346(3): 180-8.

51. American Gastroenterological Association (AGA) Institute Technical Review on the Diagnosis and Management of Celiac Disease. Gastroenterology 2006; 131: 1981-2002.

52. La Villa G, Pantaleo P, Tarquini R, Cirami L, Perfetto F, Mancuso F, et al. Multiple immune disorders in unrecognized celiac disease: a case report. World J Gastroenterol 2003; 9(6): 1377-80.

53. Ciclitira PJ, Moodie SJ. Coeliac disease. Best Prac \& Research Clin Gastroenterol 2003; 17(2): 181-95.

54. Garcia Novo MD, Garfia C, Acuña Quirós MD, Asensio J, Zancada G, Barrio Gutierrez S, et al. Prevalencia de la enfermedad celiaca en donantes de sangre de la Comunidad de Madrid. Rev Esp Enferm Dig 2007; 99: 337-42.

55. Farell RJ, Kelly CP. Celiac sprue and refractory sprue. En: Feldman M, Friedman LS, Brandt LJ, editors. Sleisenger and Fordtran's gastrointestinal and liver diseases. $8^{\text {th }}$ ed. Saunders-Elsevier; 2006. p. 2277-306

56. Carroccio A, Vitale G, Di Prima L, Chifari N, Napoli S, La Russa C, et al. Comparison of anti-transglutaminase ELISAs and an anti-endomysial antibody assay in the diagnosis of celiac disease: a prospective study. Clin Chem 2002; 48: 1546-50.

57. Macdonald WC, Brandborg LL, Flick AL, Trier JS, Rubin CE. Studies of celiac sprue. IV. The response of the whole length of the small bowel to a gluten-free diet. Gastroenterology 1964; 47: 573-89.

58. Grefte JM, Bouman JG, Grond J, Jansen W, Kleibeuker JH. Slow and incomplete histological and functional recovery in adult gluten sensitive enteropathy. J Clin Pathol 1988; 41: 886-91.
59. Tonutti E, Visentini D, Bizzaro N, Caradonna M, Cerni L, Villalta D, et al. French-Italian Laboratory Study Group on Coeliac Disease. The role of antitissue transglutaminase assay for the diagnosis and monitoring of coeliac disease: a French-Italian multicentre study. J Clin Pathol 2003; 56: 389-93.

60. Farrel RJ, Kelly CP. Diagnosis of celiac sprue. Am J Gastroenterol 2001; 96(12): 3237-46.

61. Jones B, Bayless TM, Fishman EK, Siegelman SS. Lymphadenopathy in celiac disease: computed tomographic observations. Am J Roentgenol 1984; 142: 1127-32.

62. Peña Cala MC, Gomá Masip F, Nebreda Durán J, Juan-Creix A. Linfoadenopatías mesentéricas en la enfermedad celiaca. Rev Esp Enferm Dig 2004; 96: 804-5

63. Tomei E, Diacinti D, Marini M, Mastropasqua M, Di Tola M, Sabbatella L, et al. Abdominal CT findings may suggest coeliac disease. Dig Liver Dis 2005; 37: 389-90.

64. Méndez-Uriburu L, Hawalli J, Fajre L, Ortiz Mayor M. Síndrome del ganglio linfático mesentérico cavitado. Radiologia 2006; 48: 241-4.

65. Huppert BJ, Farell MA. Case 60: cavitating mesenteric lymph node syndrome. Radiology 2003; 228: 180-4.

66. Casellas F, López Vivancos J, Malagelada JR. Epidemiología actual y accesibilidad al seguimiento de la dieta de la enfermedad celiaca del adulto. Rev Esp Enferm Dig 2006; 98: 408-19.

67. Carroccio A, Di Pima L, Scalici C, Soresi M, Cefalù AB, Noto D, et al. Unexplained elevated serum pancreatic enzymes: a reason to suspect celiac disease. Clin Gastroenterol Hepatol 2006; 4: 455-9.

68. Kim KP, Kim MH, Song MH, Lee SS, Seo DW, Lee SK. Autoimmune chronic pancreatitis. Am J Gastroenterol. 2004; 99: 1605-16.

69. Collin P, Mäki M. Associated disorders in coeliac disease: clinical aspects. Scand J Gastroenterol 1994; 29: 769-75.

70. Horiuchi A, Kawa S, Hamano H, Hayama M, Ota H, Kiyosawa K. ERCP features in 27 patients with autoimmune pancreatitis. Gastrointest Endosc 2002; 55: 494-9.

71. Hamano H, Kawa S, Horiuchi A, Unno H, Furuya N, Akamatsu T, et al. High serum IgG4 concentrations in patients with sclerosing pancreatitis. N Engl J Med 2001; 344: 732-8.

72. Barera G, Bazzigaluppi E, Viscardi M, Renzetti F, Bianchi C, Chiumello G, et al. Macroamylasemia attributable to gluten-related amylase autoantibodies: a case report. Pediatrics 2001; 107: E93.

73. Rabsztyn A, Green PH, Berti I, Fasano A, Perman JA, Horvath K. Macroamylasemia in patients with celiac disease. Am J Gastroenterol 2001; 96: 1096-100

74. Bonetti G, Serricchio G, Giudici A, Bettonagli M, Vadacca GB, Bruno R, et al. Hyperamylasemia due to macroamylasemia in adult gluten enteropathy. Scand J Clin Lab Invest 1997; 57: 271-3.

75. Pearson RK, Longnecker DS, Chari ST, Smyrk TC, Okazaki K, Frulloni L, et al. Controversies in clinical pancreatology: autoimmune pancreatitis: does it exist? Pancreas 2003; 27: 1-13.

76. Bermejo JF, Carbone J, Rodríguez JJ, Macías R, Rodríguez M, Gil J, et al. Macroamylasaemia, IgA hypergammaglobulinaemia and autoimmunity in a patiente with Down syndrome and coeliac disease. Scand J Gastroenterol 2003; 4: 445-7.

77. Ruiz del Prado MY, Olivares JL, Lázaro A, Lasierra MP. Sistema HLA. Frecuencia fenotípicas y genéticas en celíacos y controles de la misma área geográfica. Rev Esp Enferm Dig 2001; 93(2): 106-9.

78. Rostom A, Murray JA, Kagnoff MF. American Gastroenterological Association (AGA) Institute technical review on the diagnosis and management of celiac disease. Gastroenterology 2006; 131: 19812002

79. Rodrigo Sáez L. Celiac disease in the adult. Rev Esp Enferm Dig 2006; 98: 397-407.

80. Vader W, Stepniak D, Kooy Y, Mearin L, Thompson A, Van Rood $\mathrm{JJ}$, et al. The HLA-DQ2 gene dose effect in celiac disease is directly related to the magnitude and breadth of gluten-specific $\mathrm{T}$ cell reponses. Proc Natl Acad Sci USA 2003; 100: 12390-5. 